OPEN ACCESS

Edited by:

Senjie Lin,

University of Connecticut, USA

Reviewed by:

Shimshon Belkin,

Hebrew University of Jerusalem, Israel

Dave Kirchman

University of Delaware, USA

*Correspondence:

Marcelino T. Suzuki,

Sorbonne Universités, UPMC Univ

Paris 06, CNRS, Laboratoire de

Biodiversité et Biotechnologies Microbiennes (LBBM), Observatoire

Océanologique, F-66650

Banyuls-sur-Mer, France

suzuki@obs-banyuls.fr

${ }^{\dagger}$ Present address:

Thomas Riedel,

Department of Microbial Ecology

and Diversity Research, Leibniz

Institute DSMZ-German Collection of Microorganisms and Cell Cultures,

Braunschweig, Germany

Specialty section:

This article was submitted to

Aquatic Microbiology,

a section of the journal

Frontiers in Microbiology

Received: 04 February 2015

Accepted: 22 June 2015

Published: 10 July 2015

Citation:

Courties A, Riedel T, Rapaport A, Lebaron P and Suzuki MT (2015)

Light-driven increase in carbon yield is linked to maintenance in the proteorhodopsin-containing Photobacterium angustum S14. Front. Microbiol. 6:688. doi: 10.3389/fmicb.2015.00688

\section{Light-driven increase in carbon yield is linked to maintenance in the proteorhodopsin-containing Photobacterium angustum S14}

\author{
Alicia Courties ${ }^{1}$, Thomas Riedel ${ }^{2+}$, Alain Rapaport ${ }^{3,4}$, Philippe Lebaron ${ }^{2}$ and \\ Marcelino T. Suzuki ${ }^{2 *}$ \\ 'Sorbonne Universités, UPMC Univ Paris 06, CNRS, Laboratoire d'Océanographie Microbienne (LOMIC), Observatoire \\ Océanologique, Banyuls-sur-Mer, France, ${ }^{2}$ Sorbonne Universités, UPMC Univ Paris 06, CNRS, Laboratoire de Biodiversité et \\ Biotechnologies Microbiennes (LBBM), Observatoire Océanologique, Banyuls-sur-Mer, France, ${ }^{3}$ INRA-Supagro, UMR \\ MISTEA, Montpellier, France, ${ }^{4}$ INRA-INRIA, MODEMIC Team, Sophia Antipolis, France
}

A type of photoheterotrophic bacteria contain a transmembrane light-driven proton pump called proteorhodopsins (PRs). Due to the prevalence of these organisms in the upper water column of the World's Ocean, and their potential for light-driven ATP generation, they have been suggested to significantly influence energy and matter flows in the biosphere. To date, evidence for the significance of the light-driven metabolism of PR-containing prokaryotes has been obtained by comparing growth in batch culture, under light versus dark conditions, and it appears that responses to light are linked to unfavorable conditions, which so far have not been well parameterized. We studied light responses to carbon yields of the PR-containing Photobacterium angustum S14 using continuous culture conditions and light-dark cycles. We observed significant effects of light-dark cycles compared to dark controls, as well as significant differences between samples after $12 \mathrm{~h}$ illumination versus $12 \mathrm{~h}$ darkness. However, these effects were only observed under higher cell counts and lower pH associated with higher substrate concentrations. Under these substrate levels Pirt's maintenance coefficient was higher when compared to lower substrate dark controls, and decreased under light-dark cycles. It appears that light responses by $P$. angustum S14 are induced by the energetic status of the cells rather than by low substrate concentrations.

Keywords: carbon yield, Photobacterium angustum, photoheterotrophy, Pirt model, proteorhodopsin

\section{Introduction}

A type of photoheterotrophic bacteria contain a transmembrane light-driven proton pump called proteorhodopsins (PRs). These organisms were discovered over a decade ago when a metagenomic fragment belonging to the gammaproteobacterial SAR86-cluster was shown to contain a gene with homology to the bacteriorhodopsins found in Archaea (Beja et al., 2000). PR activity generates a proton motive force $(p m f)$ across the cell membrane, which has been postulated to lead to ATP generation by PR-containing prokaryotes (Beja et al., 2000). Although PR-based ATP generation

Abbreviations: PR, proteorhodopsin; $Y_{O B S}$, measured values of carbon yield; $Y_{E S T}$, carbon yield estimated from dilution rate, assuming a correlation between $Y$ and $D$. 
has not been directly measured in the environment, PRs have been shown to be widely distributed (e.g., Beja et al., 2001; de la Torre et al., 2003; Rusch et al., 2007). More significantly a number of studies mostly using real-time PCR or genomic data (as PRs are difficult to detect directly) has shown that PRcontaining prokaryotes might represent a large proportion of the total bacterioplankton of the upper water column (e.g., Rusch et al., 2007; Campbell et al., 2008; Riedel et al., 2010; Finkel et al., 2013). These high abundances, combined with the potential roles of PR-based ATP generation suggest that these organisms might have a significant impact on the ecology and biogeochemistry of marine systems. PR-based ATP generation has been hypothesized to have possible effects in growth rates (Gomez-Consarnau et al., 2007; Fuhrman et al., 2008), carbon metabolism (Fuhrman et al., 2008; Gonzalez et al., 2008), transport (Fuhrman et al., 2008), as well as starvation survival (Gomez-Consarnau et al., 2010; Steindler et al., 2011; Akram et al., 2013) among others.

Proteorhodopsin-coding genes are present in a wide taxonomic spectrum of microorganisms, particularly among environmentally abundant planktonic prokaryotes such as the alphaproteobacterial clades SAR11 (Candidatus Pelagibacter ubique; Giovannoni et al., 2005) and SAR116 (Candidatus Puniceispirillum marinus; Oh et al., 2010), the gammaproteobacterial clades SAR86 and SAR92 (Beja et al., 2000; Sabehi et al., 2004; Stingl et al., 2007a), the marine actinobacterial clade OM1 (Candidatus Actinomarinidae; Ghai et al., 2013), and the marine Euryarchaeota (Frigaard et al., 2006). PR-coding genes were also found in a number of strains belonging to groups frequently isolated from seawater such as the families Vibrionaceae (GomezConsarnau et al., 2010; Wang et al., 2012; Akram et al., 2013) and Flavobacteriaceae (e.g., Gomez-Consarnau et al., 2007; Gonzalez et al., 2008; Riedel et al., 2010).

Despite this relatively large amount of knowledge acquired for a group of bacteria discovered just over a decade ago, it is also remarkable that the significance of $\mathrm{PR}$-based phototrophy in the ecology and biogeochemistry of the oceans remains poorly understood. Of primary significance, hypothesized effects of PR to global biogeochemistry, such as light stimulation of growth and carbon yield remain controversial, since experiments with different strains are non-concordant. So far, light stimulation of growth and yields under favorable conditions has only been shown for the flavobacterial strain Dokdonia sp. MED134 (GomezConsarnau et al., 2010; Gonzalez et al., 2011; Kimura et al., 2011), where stimulation has been attributed to changes in central metabolism (Palovaara et al., 2014). However, this strain appears to represent the exception, since in all other strains tested so far, either light stimulation on growth was not observed [e.g., SAR11 (Giovannoni et al., 2005), SAR92 (Stingl et al., 2007b), Polaribacter sp. MED152 (Gonzalez et al., 2008), and Dokdonia sp. PRO95 (Riedel et al., 2010, 2013)], or effects of light exposure were only observed under unfavorable conditions, such as an enhanced survival after prolonged nutrient limitation in Vibrio sp. AND4 (Gomez-Consarnau et al., 2010), respiratory stress in Vibrio campbellii BAA-1116 (Wang et al., 2012) or under ionic stress in Psychroflexus torquis ATCC $700755^{\mathrm{T}}$ (Feng et al., 2013). It is worth to note that all these experiments were performed in batch cultures.
Combined, these previous results led Feng et al. (2013) to hypothesize that PR-based phototrophy might be important under stress conditions such as suboptimal $\mathrm{pH}$ or temperature, that divert energy from biosynthesis and growth to maintenance and survival. Currently maintenance energy can be estimated using chemostat experiments and Pirt's model. This model is an extension of Monod's (1950) classic chemostat model to which a maintenance term (maintenance coefficient, Pirt, 1965) is added. In practice the model (discussed in detail in the Supplementary materials) allows the measurement of maintenance to be calculated from a regression between the reciprocal of growth rates and the reciprocal of yields. Interestingly this model contrasts to the "textbook" notion in microbiology that microbial biomass in chemostats is independent of, or decreases with increases dilution rates, and just a product of the limiting substrate concentration and the yield on the limiting substrate.

Here we report on a study of where effects of light to PRcontaining bacterium Photobacterium angustum S14 were tested using continuous culture conditions and carbon limitation. We hypothesized that whenever the continuous cultures were to be illuminated, some ATP would to be supplied to cells by PR-based phototrophy, leading to lower respiratory requirements and to more carbon available for biosynthesis. In this condition carbon yield should increase and the expectation would be that under illuminated conditions more biomass would be present in the culture vessels. Initially, we assumed that cultures followed the classic chemostat model and that yields were independent of dilution rates, but after results showed that this was not the case, we used Pirt's theoretical framework to estimate maintenance levels and show that increases in carbon yields with light were only observed under conditions leading to higher maintenance energy levels.

\section{Materials and Methods}

\section{Bacterial Strain and Growth Conditions}

The PR-containing strain P. angustum S14 (formerly Vibrio sp. S14) was isolated in June 1981 from surface waters (1 m depth) of Botany Bay, NSW, Australia (Humphrey et al., 1983). Its genome has been sequenced (Lauro et al., 2009) and contains the entire PR-opsin and retinal biosynthetic pathway. This strain has been widely used as a model marine bacterium, as a copiotroph (Lauro et al., 2009; Williams et al., 2009) and as a model for UV resistance studies (Matallana-Surget et al., 2009).

Prior to all inoculations, the strain was pre-cultured in the dark at $25^{\circ} \mathrm{C}$ and $110 \mathrm{rpm}$ in $50 \mathrm{ml}$ of same medium as that used for continuous culture growth. Inoculation of the continuous cultures occurred at approximately after $8 \mathrm{~h}$, when pre-cultures reached an optical density at $620 \mathrm{~nm}\left(\mathrm{OD}_{620 \mathrm{~nm}}\right)$ of about 0.7 (under "high" substrate conditions defined below) and 0.4 (under "low" substrate conditions defined below), measured with a model 1200 spectrophotometer (Fisher Bioblock, France).

\section{Continuous Culture Experiments}

Changes in carbon yield $Y$ (ratio of $\mathrm{mg} \mathrm{POC}^{-1}$ in the reaction vessels/mg C in glucose $\mathrm{l}^{-1}$ in the inflow medium) of $P$. angustum 
S14 were tested in duplicate at two substrate concentrations. "High" substrate medium was prepared in artificial seawater (ASW, Eguchi et al., 1996) using glucose $(3 \mathrm{mM})$ as the sole carbon source, $\mathrm{NH}_{4} \mathrm{Cl}(9.35 \mathrm{mM})$ and $\mathrm{NaH}_{2} \mathrm{PO}_{4}(0.33 \mathrm{mM})$ as sources of $\mathrm{N}$ and $\mathrm{P}$ yielding a final molar C:N:P ratio of 54:28:1 to assure stoichiometric carbon limitation. For the "low" substrate medium, glucose, $\mathrm{NH}_{4} \mathrm{Cl}$ and $\mathrm{NaH}_{2} \mathrm{PO}_{4}$ were diluted fivefold. All continuous culture experiments were performed in Labfors Lux 3 photobioreactors (Infors HT, Switzerland). Inflow was controlled with an external peristaltic pump (Masterflex, USA) to provide fresh media. Outflow was controlled by the bioreactor's peristaltic pump and by drawing the media at a height that kept the continuous culture at a constant $1 \mathrm{~L}$ volume. Temperature of the bioreactor was set to $25^{\circ} \mathrm{C}$, aeration was provided by a vacuum pump/compressor (KNF Neuberger, Germany) with a rate of $250 \mathrm{ml}$ per minute and was stirring at $300 \mathrm{rpm}$, leading to oxygen saturation above $90 \%$ in all vessels. Fluorescent bulbs (14 W Grolux, F8WT5-GRO, Sylvania, UK) were used as light sources giving a light intensity (Photosynthetic Active Radiation) of $406.7 \mu \mathrm{E} \cdot \mathrm{m}^{-2} \cdot \mathrm{s}^{-1}$ measured in the media prior to inoculation with a model QSL irradiance meter (Biospherical Instrument Inc., USA).

Based on preliminary experiments that showed $P$. angustum S14 was inhibited by continuous illumination under certain media formulations (Courties, 2013), we measured carbon yields in duplicate vessels using $12 \mathrm{~h}$ light: $12 \mathrm{~h}$ dark cycles for each of the substrate levels [Vessels $\mathrm{H} 1$ and $\mathrm{H} 2$ ("high" substrate); Vessels L1 and L2 ("low" substrate)] and compared to yields in single controls ran in continuous darkness for each of the substrate levels [Control H ("high" substrate); Control L ("low" substrate)]. $10 \mathrm{ml}$ of the pre-culture were transferred into the bioreactor and the strain was grown in batch mode to an $\mathrm{OD}_{620 \mathrm{~nm}}$ of about 0.4 ("high" substrate condition) and to an $\mathrm{OD}_{620 \mathrm{~nm}}$ of about 0.15 ("low" substrate condition) measured as described above, at which time the feed and outflow pumps were started. For all experiments the target dilution rate was of $0.11 \cdot \mathrm{h}^{-1}$ corresponding to about a quarter of the maximum batch growth rate of $P$. angustum S14 using the "high" substrate medium. Oxygen saturation, temperature and $\mathrm{pH}$ were followed using built-in sensors of the bioreactor (Infors HT, Switzerland). Once $\mathrm{OD}_{620 \mathrm{~nm}}$ was stabilized (c.a. between 90 and $110 \mathrm{~h}$ ), triplicate samples for particulate organic carbon (POC), particulate organic nitrogen (PON) and cell counts were collected (these samples were considered as equivalent to those in the dark control), and at this stage the light was turned on. For the following six $12 \mathrm{~h}$ light: $12 \mathrm{~h}$ dark cycles, triplicate samples for $\mathrm{OD}_{620 \mathrm{~nm}}$ and cell counts were taken about every $4 \mathrm{~h}$ and samples for POC every $12 \mathrm{~h}$ (near the end of the light or dark phases). Sampling was stopped for the following five cycles and sampling was restarted at the same regime from cycles 12 and 13. For the dark controls $\mathrm{OD}_{620 \mathrm{~nm}}$, cell counts and POC sampling were taken at times equivalent to those taken in the treatments. Actual dilution rates were estimated by measuring the volume of outflow integrated over $24 \mathrm{~h}$.

\section{Batch Experiments}

In order to obtain growth characteristics of P. angustum S14 in batch mode, and in conditions equivalent to those used in the continuous culture experiment, we performed duplicate growth curves in the light $\left(406.7 \mu \mathrm{E} \cdot \mathrm{m}^{-2} \cdot \mathrm{s}^{-1}\right)$ and in the dark using the photobioreactors, the "high" substrate medium and the same parameters used in the continuous culture experiments. Growth was estimated from $\mathrm{OD}_{620} \mathrm{~nm}$ measurements as above.

The optimum $\mathrm{pH}$ for growth was tested in duplicate using the "high" substrate medium to which buffers MES (SigmaAldrich; for $\mathrm{pH}$ 5.5-6.5), HEPES (Sigma-Aldrich; for $\mathrm{pH} 7.0-8.0$ ), or AMPSO (Sigma-Aldrich; for $\mathrm{pH}$ 8.5-10.0) were added at $2.0 \mathrm{mg} / \mathrm{ml}$. $\mathrm{pH}$ was adjusted to a 5.5-10.0 range using $25 \%(\mathrm{v} / \mathrm{v})$ concentrated $\mathrm{HCl}$ or $1 \mathrm{~N} \mathrm{NaOH}$. Growth was followed in 24 well plates (Evergreen Scientific, USA) incubated at $25^{\circ} \mathrm{C}$ in the dark at $100 \mathrm{rpm}$. $\mathrm{OD}_{620 \mathrm{~nm}}$ measurements were obtained using a Paradigm $^{\text {TM }}$ Multi-mode Detection Platform (Molecular Devices, USA).

\section{POC and PON Measurements}

Particulate organic carbon and PON were measured from triplicate samples using a standard protocol: a volume of $5 \mathrm{ml}$ ("high" substrate experiments) and $10 \mathrm{ml}$ ("low" substrate experiments) were filtered through glass fiber filters $(\mathrm{GF} / \mathrm{F}$, Whatman, UK), which were finally placed in glass vials, dried for $8 \mathrm{~h}$, and acidified with concentrated $\mathrm{HCl}$ for at least $8 \mathrm{~h}$ and dried again. All used filters as well as glass vials had been precombusted at $450^{\circ} \mathrm{C}$ for at least $12 \mathrm{~h}$. Carbon and nitrogen was measured with a Series II $2400 \mathrm{CHNS} / \mathrm{O}$ Analyzer (Perkin-Elmer, USA) using a modified Dumas method (Jacobs, 1965). Final POC and PON values were subject to an outlier analysis where any of the triplicate samples whose value was higher than twofold or lower than 0.5 of the standard deviation of the remaining two samples was considered as an outlier. These values were removed from the analysis.

\section{FACS and Optical Density Measurements}

Triplicate $1 \mathrm{ml}$ samples were fixed with $1 \%$ glutaraldehyde (SigmaAldrich, USA), flash frozen in liquid nitrogen and stored at $-80^{\circ} \mathrm{C}$. Samples were diluted 100 -fold and stained for $15 \mathrm{~min}$ with a 1:400 dilution of SYBR green I (Molecular Probes, USA). Cell counts were measured in triplicate by flow cytometry using a FACSCalibur flow cytometer (Becton Dickinson, USA) equipped with an air-cooled argon laser $(488 \mathrm{~nm}, 15 \mathrm{~mW})$ as previously described (Van Wambeke et al., 2011).

Final $\mathrm{OD}_{620 \mathrm{~nm}}$ measurements (shown in all figures, except for Figure 1) were taken in triplicate in 96 well microplates from the same fixed samples using a Paradigm ${ }^{\mathrm{TM}}$ Multi-mode Detection Platform (Molecular Devices).

\section{Glucose Measurements}

Residual glucose in the medium was measured in triplicate (filtrate of the filters used for POC measurements) using a Glucose Assay kit (Sigma-Aldrich) adapted to a 48 well-microplate format with $\mathrm{OD}_{540 \mathrm{~nm}}$ measured using a Paradigm ${ }^{\mathrm{Tm}}$ Multi-mode Platform (Molecular Devices). Since, as expected, all measurements from experimental Vessels $\mathrm{H} 1$ were in the micromolar range, thus negligible compared to the concentration of the inflow medium, 


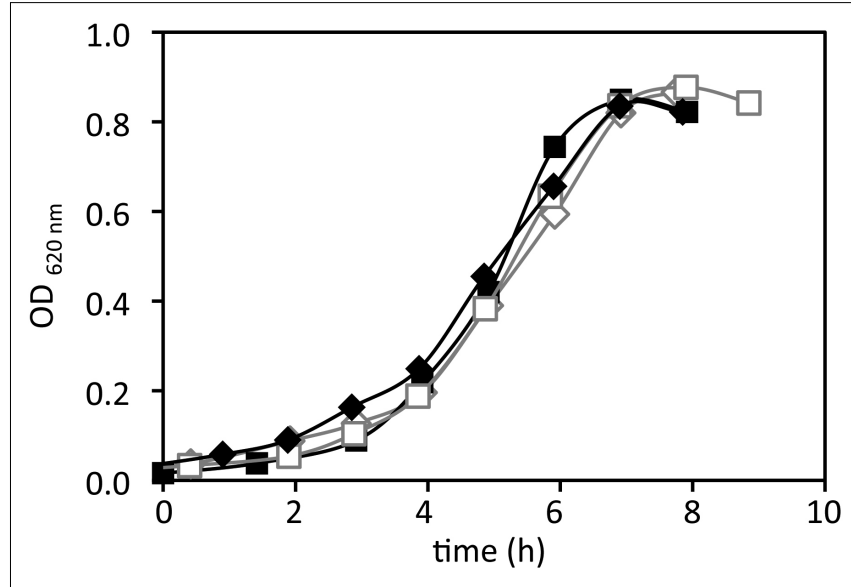

FIGURE 1 | Growth in batch of $P$. angustum $S 14$ in photobioreactor batch experiments using "high" substrate conditions. Duplicates growth in dark (black curves) and in the light $\left(406.7 \mu \mathrm{E} \cdot \mathrm{m}^{-2} \cdot \mathrm{s}^{-1}\right.$, gray curves).

a subset of samples was measured in triplicate (dark and light samples in cycle 13, and the last sample for the controls) for the remaining experiments.

\section{Data Analyses}

Statistical analyses were performed using the $\mathrm{R}^{1}$ software. Estimation of values of the maintenance coefficient, as well as the modeling used to evaluate quasi steady state conditions was scripted and ran using scilab ${ }^{2}$. Details of these analyses are presented in the supplementary methods.

\section{Results}

In order to evaluate the effect of light on growth of $P$. angustum S14 in batch and estimate growth parameters, batch experiments were run in photobioreactors with the same medium, substrate, agitation and aeration as those used in continuous culture experiments at "high" (i.e., $3 \mathrm{mM}$ glucose) substrate levels. In these conditions $P$. angustum $\mathrm{S} 14$ grew at a rate of approximately $0.4 \mathrm{~h}^{-1}$ and the growth rates were not significantly different (ANCOVA, $p=0.71)$ between illuminated $\left(0.442 \pm 0.047 \mathrm{~h}^{-1}\right)$ and dark $\left(0.468 \pm 0.047 \mathrm{~h}^{-1}\right)$ conditions (Figure 1).

\section{Continuous Culture Experiments: "High" Substrate Levels}

The first set of continuous cultures was run on what we called "high" substrate levels. The progression of biomass in two replicate continuous cultures (Vessel $\mathrm{H} 1$ and Vessel H2) under $12 \mathrm{~h}$ light and $12 \mathrm{~h}$ dark cycles, and in a control continuous culture kept in the dark (Control H), is shown in Figure 2. We observed that after about $80 \mathrm{~h}$, the $\mathrm{OD}_{620 \mathrm{~nm}}$ and cell counts (Figure S1 in supplementary material) were relatively stable (within $\pm 0.5 \times$ of the average values) except in Vessel $\mathrm{H} 1$ where $\mathrm{OD}_{620 \mathrm{~mm}}$ and cell counts increased with a corresponding decrease in $\mathrm{pH}$ at ca. $140 \mathrm{~h}$, a time corresponding to a new batch of medium being used

${ }^{1}$ http://www.r-project.org

${ }^{2}$ https://www.scilab.org/
(Figure S1 in supplementary material; arrow). In all vessels the $\mathrm{pH}$ was low (ca. 5.0-6.0) when compared to the optimum $\mathrm{pH}$ for growth of $P$. angustum S14 (pH 8.0-9.0; data not shown) reflecting the relatively high cell counts (ca. 0.5-1.0 $10^{9}$ cells $\cdot \mathrm{ml}^{-1}$ ). Throughout 13 light-dark cycles all parameters were relatively stable in both replicates as well as in the control. We observed nonetheless a slight increase in POC, and thus carbon yield, throughout the entire experiment for Vessel H1, as well as some increases after $12 \mathrm{~h}$ illumination, particularly during cycles 12 and 13 in Vessel H1 and during early cycles in Vessel H2 (Figure 2). We also observed clear drops (ca. 0.1-0.2) in C:N ratios after $12 \mathrm{~h}$ of illumination in both illuminated vessels (Figure 3 ). In these conditions both POC and PON increased but relatively PON increased more, leading to a drop of the ratio (Figure S2 in supplementary material). These variations were not observed in the control vessel (Figure 3). Treating each light-dark cycle as a replicate measure, this decrease was very significant (ANOVA, $p<0.001)$ for Vessel H1 and significant $(p=0.022)$ for Vessel $\mathrm{H} 2$ after cycle 4 . Glucose concentrations in all measured samples were in the micromolar range (about 1:1000 than that of the inflow media), and thus it was assumed to be 0 in subsequent calculations.

One remarkable observation was that the average POC values, and thus $Y$ (expressed in \% hereafter), were significantly higher (ANOVA, $p<0.001)$ in the dark control $(56.1 \pm 2.25)$, than either of the treatment Vessels $[46.8 \pm 0.003(\mathrm{H} 1)$ and $48.2 \pm 0.015$ (H2)] and very importantly, this difference existed even when only comparing values prior to the start of light-dark cycles (Figure 2). Apart from the obvious difference in light regimes between the treatment vessels and the control, we observed that the dilution rates were not maintained perfectly constant during experiments (Figure S3 in supplementary material). Variations in dilution led the continuous culture to a sequence of quasi-steady states that allowed measurements under different dilutions.

We used Pirt's theoretical framework (Supplementary Methods) and estimated $m$ values from a regression line between $1 / Y$ and $1 / \mu$, assuming the system was at steady state (i.e., $\mu=D$, the dilution rate). To evaluate the maintenance coefficient under different conditions, regressions parameters were calculated for $Y$ values measured in continuous darkness (in both replicates and in the control; $\mathrm{R}_{D D}$ ), $Y$ values measured after $12 \mathrm{~h}$ illumination $\left(\mathrm{R}_{L C}\right)$, and $Y$ values measured after $12 \mathrm{~h}$ darkness $\left(\mathrm{R}_{D C}\right)$. Since $D$ was measured at different times at a lower frequency than $Y$, three regressions lines were calculated using three different values of $D$ [prior $\left(\mathrm{R}_{D D-P}, \mathrm{R}_{L C-P}, \mathrm{R}_{L C-P}\right)$, after the $Y$ measurement $\left(\mathrm{R}_{D D-A}, \mathrm{R}_{L C-A}, \mathrm{R}_{L C-A}\right)$, and using interpolated $D$ values $\left.\left(\mathrm{R}_{D D-I}, \mathrm{R}_{L C-I}, \mathrm{R}_{L C-I}\right)\right]$. The results (Figure 4) show that $m$ was higher $\left(0.142 \pm 0.020 \mathrm{~h}^{-1}\right)$ when $Y$ was measured in continuous darkness (Figure 4A) than both when $Y$ was measured after $12 \mathrm{~h}$ illumination $\left(m=0.005 \pm 0.036 \mathrm{~h}^{-1}\right.$; Figure 4B) or after $12 \mathrm{~h}$ darkness $\left(m=0.007 \pm 0.040 \mathrm{~h}^{-1}\right.$; Figure 4C), suggesting that light-dark cycles might have led to a decrease of the maintenance coefficient of $P$. angustum S14.

Since we observed an effect of varying dilution on yields, we performed a correction of $Y$ before testing if light-dark cycles led to differences in carbon yields. This correction removed the 


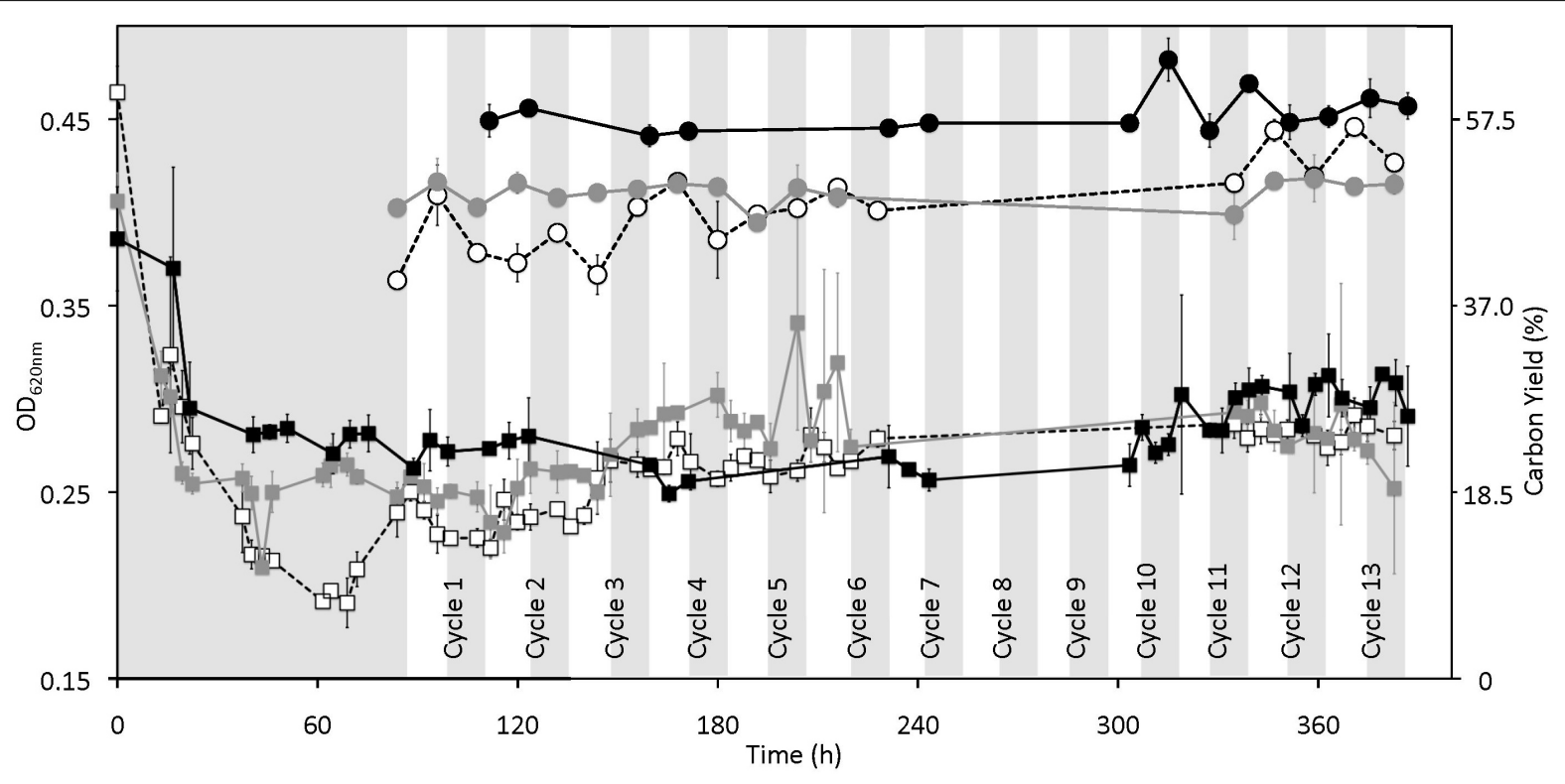

FIGURE 2 | Effect of cyclic $12 \mathrm{~h}$ illumination and $12 \mathrm{~h}$ darkness on the biomass and carbon yield of $P$. angustum $\mathbf{S} 14$ grown in chemostats under "high" substrate conditions. Gray and white vertical bars represent dark and light periods respectively for treatment chemostats, onto which data from the dark-only control were plotted based on sampling time. White squares, gray squares and black squares represent $\mathrm{OD}_{620 \mathrm{~mm}}$ values for Vessel H1, Vessel H2, and Control H, respectively. Open circles with dotted lines, gray circles with gray lines and black circles with black lines represent carbon yield in percentage for Vessel $\mathrm{H} 1$, Vessel $\mathrm{H} 2$, and Control $\mathrm{H}$ respectively.

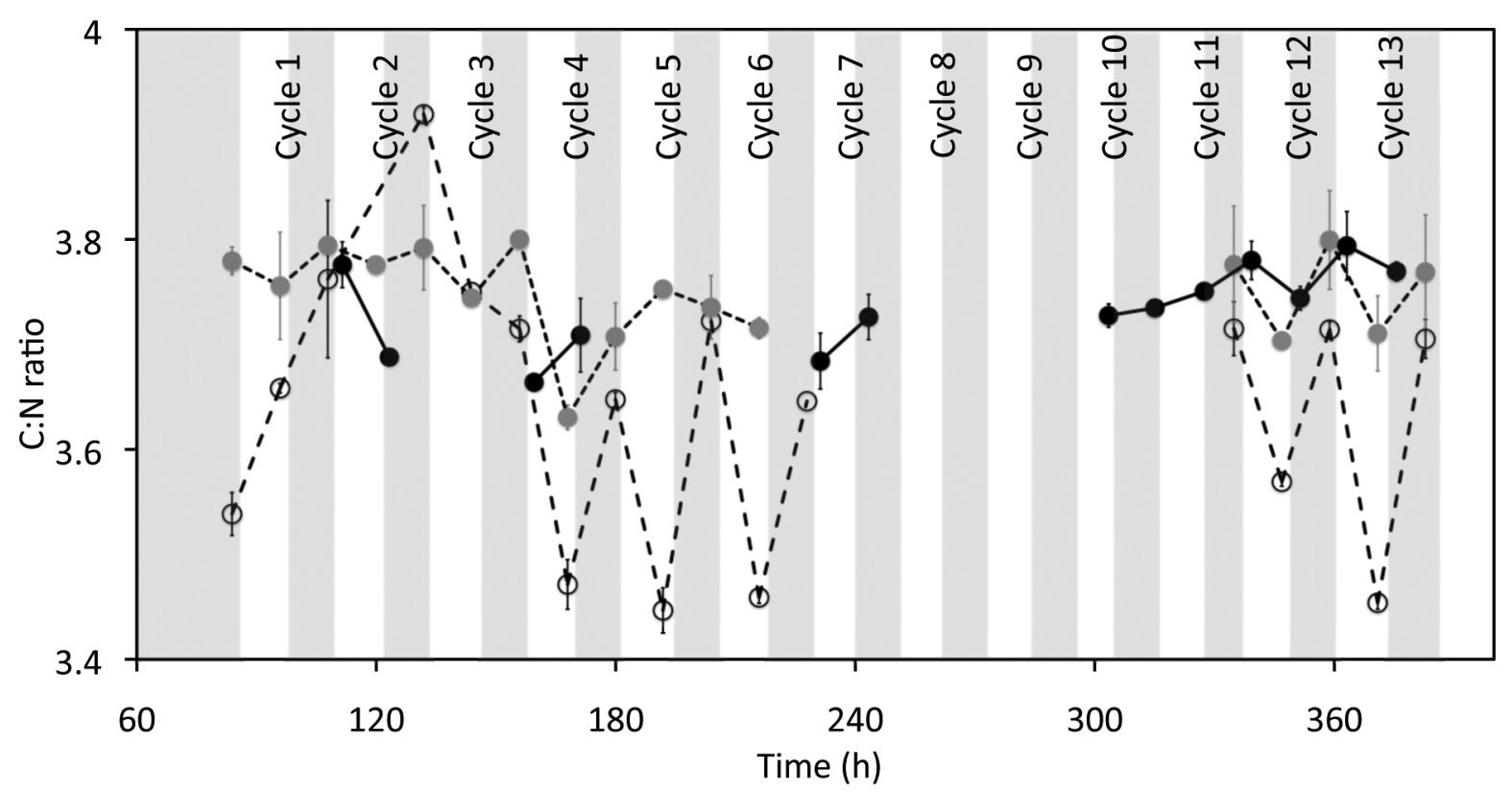

FIGURE 3 | Effect of cyclic $12 \mathrm{~h}$ illumination and $12 \mathrm{~h}$ darkness on the C:N ratio of $P$. angustum S14 cells grown in chemostats under "high" substrate conditions. Vertical bars are as in Figure 2. White circles, gray circles and black circles represent $\mathrm{C}: \mathrm{N}$ ratios for Vessel $\mathrm{H} 1$, Vessel H2, and Control $\mathrm{H}$, respectively.

confounding effect of variations dilution rates. We estimated what we called expected yields $\left(Y_{E S T}\right)$ from interpolated $D$ values using regression $\mathrm{R}_{D D-I}$ (black diamonds in Figure $4 \mathrm{~A}$ ). These values were then subtracted from the measured $Y$ values $\left(Y_{O B S}\right)$ yielding an anomaly $\left(Y_{O B S}-Y_{E S T}\right)$ that allowed a comparison of yields under light and dark conditions, independent of variations in $D$
(Figure 5). It is clear that this anomaly was significantly lower for samples taken in continuous darkness $(0.23 \pm 3.71)$ than for samples taken after $12 \mathrm{~h}$ illumination or after $12 \mathrm{~h}$ darkness (ANOVA; post hoc Tukey's test; $p<0.001$ ). The anomaly was not significantly different between samples taken after $12 \mathrm{~h}$ of illumination $(6.91 \pm 4.21)$ and those taken after $12 \mathrm{~h}$ of darkness 


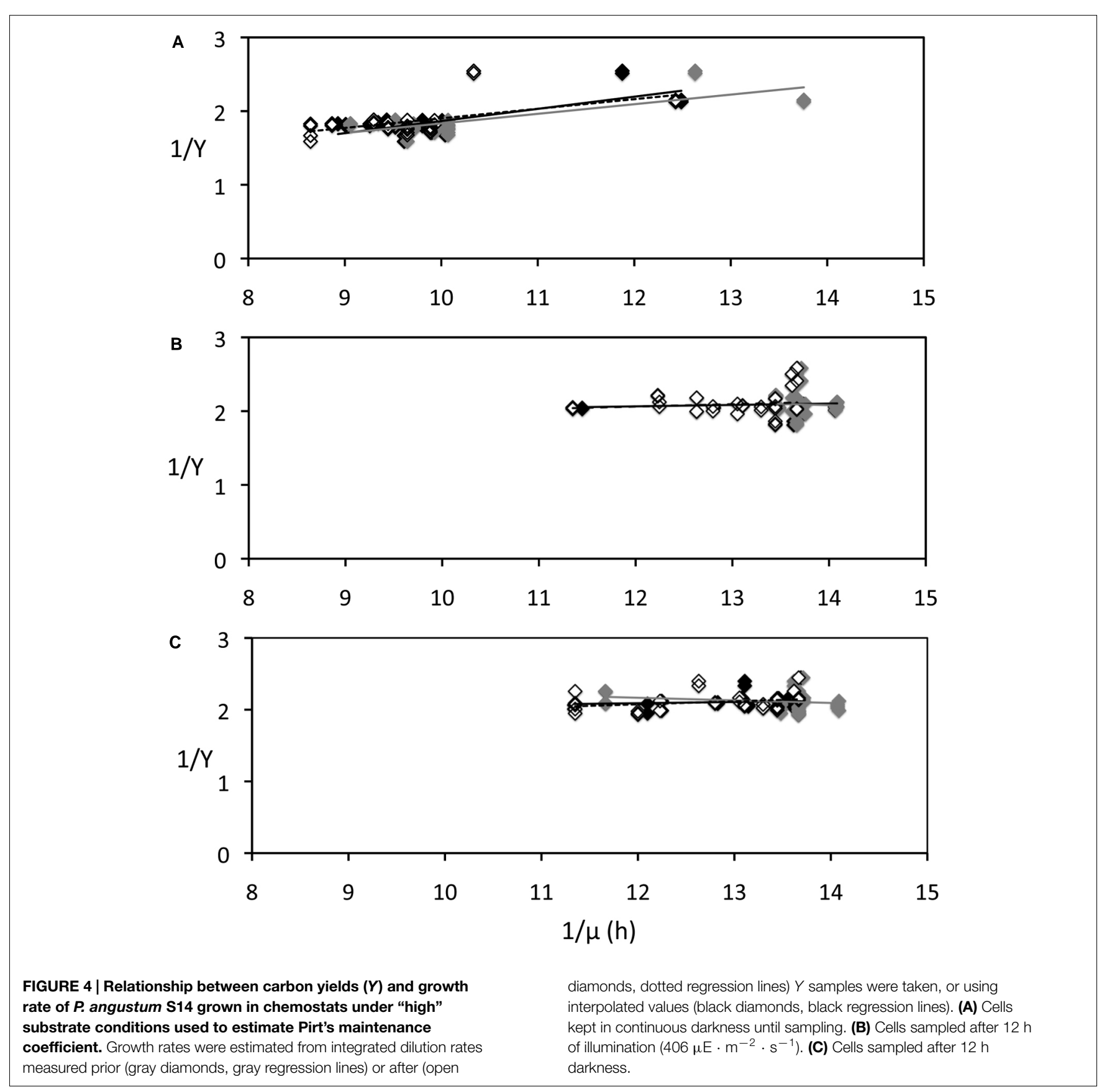

$(5.05 \pm 3.14)$ based on the ANOVA post hoc Tukey test $(p=0.10)$, but was significantly higher $(p=0.043)$ using a pairwise $t$ test.

\section{Continuous Culture Experiments: "Low" Substrate Levels}

A second set of experiments was run at what we called "low" substrate levels (600 $\mu \mathrm{M}$ glucose, same C:N:P ratio). As for the "high" substrate levels experiments, two replicate continuous cultures under 12-12 cycles (Vessel L1 and Vessel L2) and a dark control continuous culture (Vessel L) were performed. Contrary to our expectation, we did not observe the same effects of light on carbon yields and stoichiometry of P. angustum S14 under a lower level of substrate addition. $\mathrm{OD}_{620} \mathrm{~nm}$ values were more scattered compared to the "high" substrate, particularly for the illuminated bioreactors, but this was the case even before the light was turned on (Figure S4 in supplementary material). Throughout the cycles a very slight decrease in $\mathrm{OD}_{620 \mathrm{~nm}}$, also reflected in a decrease POC values was observed. C:N ratios showed a steady decrease after illumination arriving at a value of about 3.4 in illuminated bioreactors, but there were no clear light-dark variations such as those observed in the "high" 


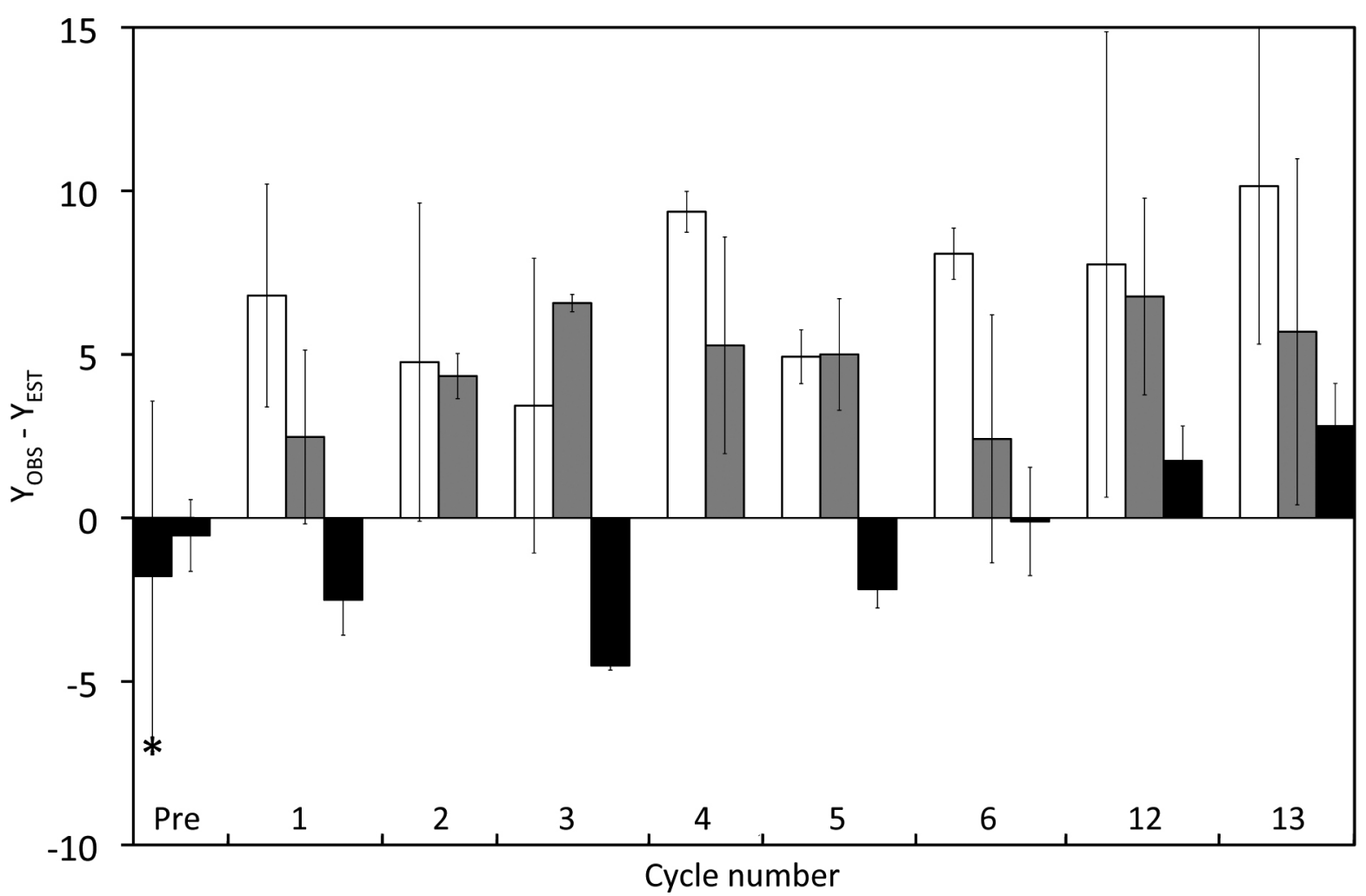

FIGURE 5 | Effect of cyclic $12 \mathrm{~h}$ illumination $\left(406.7 \mu \mathrm{E} \cdot \mathrm{m}^{-2} \cdot \mathrm{s}^{-1}\right)$ and $12 \mathrm{~h}$ darkness on the carbon yield of $P$. angustum $\mathrm{S} 14$ grown in chemostats under "high" substrate conditions. The $Y_{O B S}-Y_{E S T}$ anomaly corresponds to $Y$ values corrected for the effect on $Y$ of varying dilution rates.
Dark bars: Cells kept in continuous darkness until sampling. Light bars: Cells sampled after $12 \mathrm{~h}$ of illumination. Gray Bars: Cells sampled after $12 \mathrm{~h}$ darkness. The asterisk corresponds samples from Vessel $\mathrm{H} 1$ and Vessel $\mathrm{H} 2$, prior to the start of light-dark cycles. substrate experiments (Figure S5 in supplementary material). Levels of $\mathrm{pH}$ were much higher (Figure S6 in supplementary material) than those in "high" substrate experiments (Figure S1 in supplementary material), very likely due to the lower cells numbers (Figure S6 in supplementary material). These lower cell numbers and higher $\mathrm{pH}$ might partly explain the significantly higher $Y$ values in the "low" substrate experiments (mean of controls and treatments 62.01 \pm 2.73 ) compared to "high" substrate experiments (mean of controls and treatments $49.96 \pm 5.02$; ANOVA, $p<0.001)$.

We calculated the maintenance coefficient in the same way as in the "high" substrate experiments (Figure S7 in supplementary material), and $m$ values were low, as $Y$ appears to be nearly independent of $\mu$ for the dark samples $\left(m=0.019 \pm 0.007 \mathrm{~h}^{-1}\right)$ as well as samples collected after $12 \mathrm{~h}$ illumination $\left(m=0.011 \pm 0.001 \mathrm{~h}^{-1}\right)$ or after $12 \mathrm{~h}$ darkness $\left(m=-0.018 \pm 0.008 \mathrm{~h}^{-1}\right)$. For comparison sake we calculated the anomaly $\left(Y_{O B S}-Y_{E S T}\right)$ shown in Figure 6. Except for one value, all anomaly values from Vessels L1 and L2 were negative and $Y_{O B S}-Y_{E S T}$ calculated from Vessels L1 and L2 were significantly different to those in Control L shown by an ANOVA $(p<0.001)$ and a pairwise $t$-test $(p \leq 0.001)$.

\section{Discussion}

As previously shown for the other members of the family Vibrionaceae (Gomez-Consarnau et al., 2010; Wang et al., 2012; Akram et al., 2013), P. angustum S14 shows no responses to light under "normal" batch growth, and this is becoming the norm for these organisms, whereas the picture is more complicated for the Flavobacteriaceae even when the same genus is considered (Gomez-Consarnau et al., 2007; Riedel et al., 2013). The reasons for this discrepancy remain elusive and thus the examination of another strain with a sequenced genome such as $P$. angustum S14 will provide additional data to studies of comparative genomics targeting this question.

In addition, our initial working model assumed that $P$. angustum S14 cells would respond to substrate limitation in continuous cultures and modify their metabolism to use light as a supplementary energy source. This assumption did not appear to hold for this PR-containing prokaryote. This is in contrast to recent studies that measured carbon yields of the anoxygenic aerobic photoheterotroph Erythrobacter sp. NAP1 using continuous cultures (Hauruseu and Koblizek, 2012) which observed quite large differences in yield under illuminated 


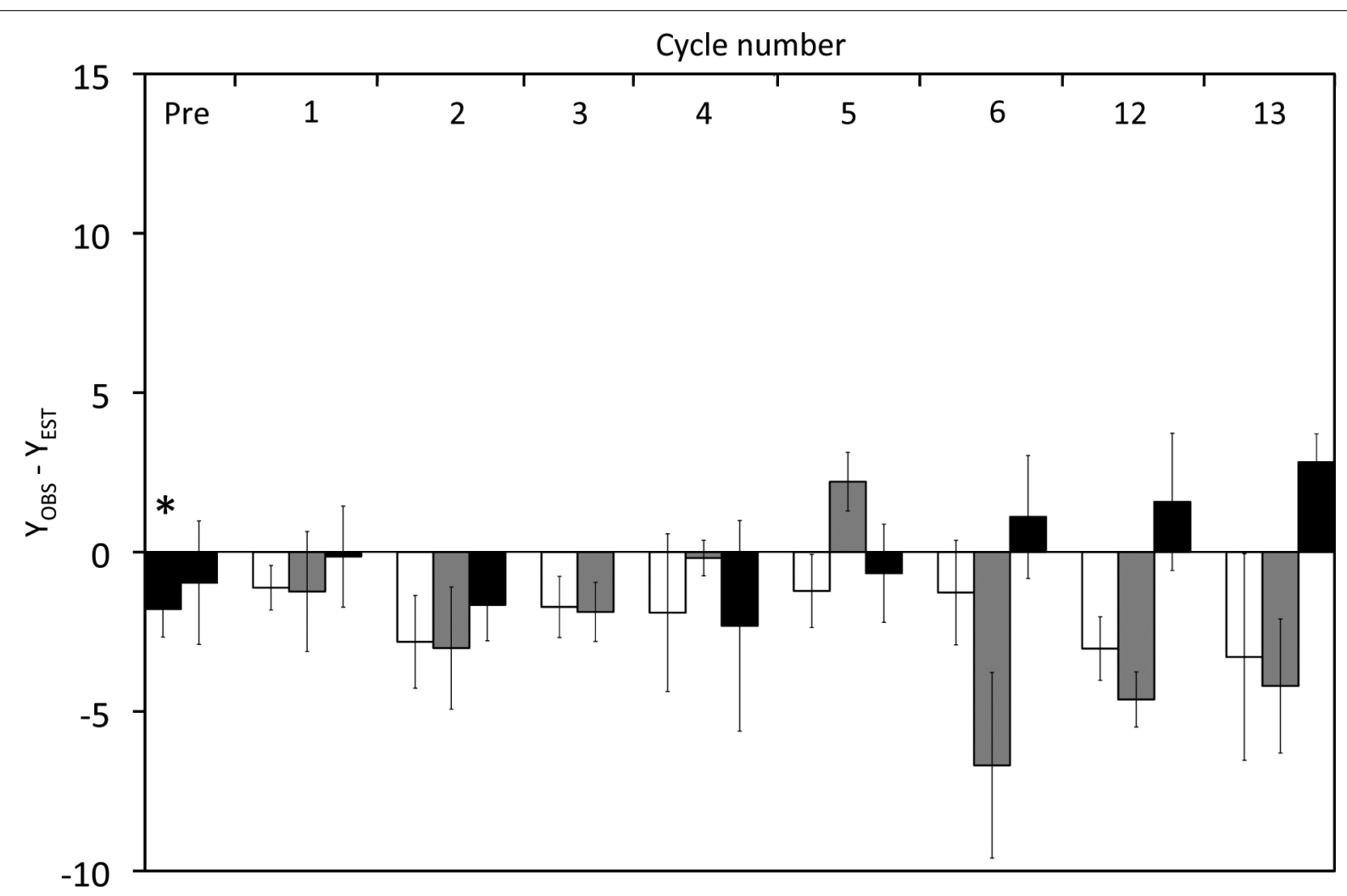

FIGURE 6 | Effect of cyclic $12 \mathrm{~h}$ illumination and $12 \mathrm{~h}$ darkness on the carbon yield of $P$. angustum $\mathrm{S} 14$ grown in chemostats under "low" substrate conditions. The $Y_{O B S} Y_{E S T}$ anomaly corresponds to $Y$ values corrected for the effects of dilution rates on $Y$. Back bars: Cells kept in continuous darkness until sampling. White bars: Cells sampled after $12 \mathrm{~h}$ of illumination. Gray Bars: Cells sampled after $12 \mathrm{~h}$ darkness. The asterisk corresponds samples from Vessel L1 and Vessel L2, prior to the start of light-dark cycles. conditions (dark and light cycles), and in agreement with previous theoretical predictions (Kirchman and Hanson, 2013). It appears that, at least for $P$. angustum S14, responses in $Y$ to light are not solely dependent on carbon limitation. While it might be argued that the $3 \mathrm{mM}$ of glucose is a high concentration relative to environmental conditions, high concentrations were needed to yield enough biomass (POC) that could be measured in a small enough volume that would not significantly interfere with the work volume of the continuous cultures. Furthermore, it is important to point out that residual glucose in the bioreactors were measured in micromolar concentrations, and that the C:N ratios were low, indicating that these cells were very likely carbon limited.

A number of responses to light (or to dark-light cycling) were observed in the "high" substrate bioreactor experiments that either did not occur, or were different to the "low" substrate bioreactor experiments. We observed significant increases of the $Y_{O B S}-Y_{E S T}$ anomaly (ca. 6.0\%) after light-dark cycles compared to full darkness, which were not observed in the "low" substrate experiments. This anomaly represents an increase in yield of about $15 \%$ relative to the $Y_{E S T}$ values calculated from dilution rates. However, since dilutions and yields were clearly higher in dark controls, and the slopes of the regression in Figure 4A were strongly influenced by pre-cycle values of Vessels $\mathrm{H} 1$ and
$\mathrm{H} 2$, these values should be viewed conservatively. A second observed response only seen in "high" substrate was that in several cycles, we observed higher $Y_{O B S}-Y_{E S T}$ after $12 \mathrm{~h}$ of illumination compared to the previous or subsequent $12 \mathrm{~h}$ in darkness, and the differences between $Y_{O B S}-Y_{E S T}$ after $12 \mathrm{~h}$ illumination or after $12 \mathrm{~h}$ darkness were significant, albeit only for the $t$-test. Again, these differences were not observed in the "low" substrate experiment. Finally, very clear and significant decreases in C:N with illumination only seen in the "high" substrate experiments suggests a change in the physiology of $P$. angustum S14 with light, possibly leading to an increase in protein content relative to other cellular biomolecules with light.

Thus, it is clear that most of $P$. angustum S14 responses to light or light-dark cycles happened only under higher substrate levels that yielded higher biomass and led to lower $\mathrm{pH}$ levels. While we cannot unequivocally attribute these responses to $\mathrm{pH}$ or any specific factor, both the lower $Y$ values in "high" substrate bioreactor experiments, as well as estimates of $m$ from dark controls and pre-cycle samples, point to a higher cost of maintenance in these bioreactors compared to those in the "low" substrate additions, and in agreement with the suggestions by Feng et al. (2013) based on an analysis of several previous studies, that PR (thus light) can have an effect in conditions 
leading to "diversion of energy from growth and biosynthesis to maintenance and survival functions." Since the original range of dilution rates used to calculate $m$ was narrow, we performed additional experiments ("high" substrate, dark only) to measure $m$ at a wider range of dilution rates. Interesting, while calculated $m$ values were similar for $D$ in the $0.075-0.1 \mathrm{~h}^{-1}$ range, lower $D$ values $\left(0.05-0.02 \mathrm{~h}^{-1}\right)$ yielded lower biomass and higher $(>6.0) \mathrm{pH}$ levels. Values of $m$ were clearly lower for those samples further supporting the effects of $\mathrm{pH}$ on maintenance.

Finally photoinhibition such as that observed in V. campbellii BAA-1116 (Wang et al., 2012) could be suggested as an alternative explanation to the observed lower values of yields in illuminated continuous cultures compared to those in the control, but this seems unlikely the case since (1) lower POC (thus $Y$ ) were observed previous to the first cycle, (2) POC (thus $Y$ ) increased or stayed relatively stable after 13 cycles, while a decrease would be expected under photoinhibition, (3) the anomaly $Y_{O B S}-Y_{E S T}$ was always higher in illuminated continuous cultures when compared to the control, and (4) inhibition of growth rates would have led to relatively higher residual substrate at steady state than we observed.

We did not directly measure the expression of the PR-coding gene or of genes involved in the retinal biosynthetic pathway and thus we cannot assign these effects to PR proton pumping, but this remains as one of the most parsimonious explanations. The light levels used in our experiments were high compared to most of the previous experiments (Giovannoni et al., 2005; Gomez-Consarnau et al., 2007, 2010; Kimura et al., 2011; Wang et al., 2012; Akram et al., 2013; Feng et al., 2013; Riedel et al., 2013), and were chosen based on preliminary experiments (albeit with a different media and continuous light) showing that lower light levels $\left(224 \mu \mathrm{mol}\right.$ of photons $\left.\cdot \mathrm{m}^{-2} \cdot \mathrm{s}^{-1}\right)$ did not elicit responses that were observed at $406.7 \mu \mathrm{E} \cdot \mathrm{m}^{-2}$ $\cdot \mathrm{s}^{-1}$. Assuming that PR activity mediated the observed light responses by $P$. angustum S14, since this bacterium was originally isolated from surface waters, responses under these high light levels is not unexpected. Additionally, the PR of P. angustum S14 is predicted to be mainly a blue-absorbing PR based on the presence of a glutamine at spectral tuning position 105 (EBAC31A08 numbering). Blue-absorbing PRs retrieved from the Ocean surface were shown to have faster photocycles, indicative of adaptation to higher irradiances (Sabehi et al., 2005), even though this is not true for blue-absorbing PRs retrieved from deeper in the water column (e.g., Beja et al., 2001; Wang et al., 2003).

If confirmed in other PR-containing prokaryotes, the relationship between the maintenance coefficient and light responses by $P$. angustum S14 could be used to evaluate whether light is actually playing a role in PR-containing prokaryote metabolism in the environment, based on measurements of the maintenance coefficient of naturally occurring bacterioplankton under different light conditions. To date, few studies have attempted to estimate maintenance costs of mixed bacterioplankton assemblages (Cajal-Medrano and Maske, 1999, 2005). In these studies measurements were made for entire communities, using much lower dilution rates and levels of carbon addition, complicating comparisons to our data. In the latter study (Cajal-Medrano and Maske, 2005) measured $m$ values $\left(0.33 \mathrm{~h}^{-1}\right)$ were higher than those we measured for $P$. angustum S14 and assuming these values are the same for PR-containing prokaryotes and other microorganisms, light-driven metabolism could contribute to fulfill maintenance requirements of PRcontaining prokaryotes in the environment. The combination of molecular identification using probes and possible single cell estimates of respiration in dark versus light conditions, if developed, could provide better estimates of the contribution of light specifically to the metabolism of PR-containing prokaryotes in the future.

Finally our results suggest that unlike Dokdonia sp. MED134 (Gomez-Consarnau et al., 2007) responses to light by P. angustum S14 do not appear to be inversely correlated to substrate levels or limitation, but rather to the overall energetic balance of the cells, and thus again assuming these responses are caused by $\mathrm{PR}$ activity, biochemical mechanisms sensing energy levels rather than substrate levels might be mostly involved in the regulation of production and/or activity of PR-opsin together with its cofactor retinal. Future transcriptomic or proteomic studies with $P$. angustum S14 could lead to a better understanding of this regulation.

\section{Author Contributions}

MS, AC, and PL designed the experiment. AC performed or significantly participated in all of the experimental work. TR performed most glucose analysis as well as OD measurements and POC analysis. AC, AR, and MS were responsible of continuous culture modeling and statistical analysis. AC, TR, MS, AR, and PL wrote the manuscript, approved its final version and agree to be accountable for all aspects of the work.

\section{Acknowledgments}

This work was supported by the French ANR project RHOMEO (ProteoRHOdopsin-containing prokaryotes in Marine EnvirOnments) and a doctoral fellowship from the UPMC ED392 to Alicia Courties. We thank Maria C. Nielsdóttir, Nicole Batailler, Maria Papadatou, and Marie Olloix for assistance with continuous culture experiments. We thank Jocelyne Caparros for POC analyses. We thank Philippe Catala and Christophe Salmeron for Flow Cytometry analyses. We thank Mathilde Fauvin for conducting the additional $m$ measurement experiment. We also thank Ingrid Obernosterer and Stéphane Blain for fruitful discussions, and members of the MODEMIC team (Jerome Harmand, Fabien Campillo, Claude Lobry, and Tewif Sari) for discussions regarding chemostat theory and modeling.

\section{Supplementary Material}

The Supplementary Material for this article can be found online at: http://journal.frontiersin.org/article/10.3389/fmicb.2015.00688 


\section{References}

Akram, N., Palovaara, J., Forsberg, J., Lindh, M. V., Milton, D. L., Luo, H., et al. (2013). Regulation of proteorhodopsin gene expression by nutrient limitation in the marine bacterium Vibrio sp. AND4. Environ. Microbiol. 15, 1400-1415. doi: 10.1111/1462-2920.12085

Beja, O., Aravind, L., Koonin, E. V., Suzuki, M. T., Hadd, A., Nguyen, L. P., et al. (2000). Bacterial rhodopsin: evidence for a new type of phototrophy in the sea. Science 289, 1902-1906. doi: 10.1126/science.289.5486.1902

Beja, O., Spudich, E. N., Spudich, J. L., Leclerc, M., and DeLong, E. F. (2001). Proteorhodopsin phototrophy in the ocean. Nature 411, 786-789. doi: $10.1038 / 35081051$

Cajal-Medrano, R. N., and Maske, H. (1999). Growth efficiency, growth rate and the remineralization of organic substrate by bacterioplankton: revisiting the Pirt model. Aquat. Microb. Ecol. 19, 119-128. doi: 10.3354/ame019119

Cajal-Medrano, R. N., and Maske, H. (2005). Growth efficiency and respiration at different growth rates in glucose-limited chemostats with natural marine bacteria populations. Aquat. Microb. Ecol. 38, 125-133. doi: 10.3354/ ame038125

Campbell, B. J., Waidner, L. A., Cottrell, M. T., and Kirchman, D. L. (2008). Abundant proteorhodopsin genes in the North Atlantic Ocean. Environ. Microbiol. 10, 99-109. doi: 10.1111/j.1462-2920.2007.01436.x

Courties, A. (2013). Les effets de la lumière sur le métabolisme du carbone des bactéries marines contenant la protéorhodopsine: cas détude en culture continue d'une Gammaprotéobactérie Photobacterium angustum S14. Ph.D. thesis, Sous la direction de Philippe Lebaron et Marcelino Suzuki, Paris 6.

de la Torre, J. R., Christianson, L. M., Beja, O., Suzuki, M. T., Karl, D. M., Heidelberg, J., et al. (2003). Proteorhodopsin genes are distributed among divergent marine bacterial taxa. Proc. Natl. Acad. Sci. U.S.A. 100, 12830-12835. doi: $10.1073 /$ pnas. 2133554100

Eguchi, M., Nishikawa, T., Macdonald, K., Cavicchioli, R., Gottschal, J. C., and Kjelleberg, S. (1996). Responses to stress and nutrient availability by the marine ultramicrobacterium Sphingomonas sp. Strain RB2256. Appl. Environ. Microbiol. 62, 1287-1294.

Feng, S., Powell, S. M., Wilson, R., and Bowman, J. P. (2013). Light-stimulated growth of proteorhodopsin-bearing sea-ice psychrophile Psychroflexus torquis is salinity dependent. ISME J. 7, 2206-2213. doi: 10.1038/ismej. 2013.97

Finkel, O. M., Béjà, O., and Belkin, S. (2013). Global abundance of microbial rhodopsins. ISME J. 7, 448-451. doi: 10.1038/ismej.2012.112

Frigaard, N. U., Martinez, A., Mincer, T. J., and DeLong, E. F. (2006). Proteorhodopsin lateral gene transfer between marine planktonic bacteria and Archaea. Nature 439, 847-850. doi: 10.1038/nature04435

Fuhrman, J. A., Schwalbach, M. S., and Stingl, U. (2008). Proteorhodopsins: an array of physiological roles? Nat. Rev. Microbiol. 6, 488-494. doi: 10.1038/nrmicro1893

Ghai, R., Mizuno, C. M., Picazo, A., Camacho, A., and Rodriguez-Valera, F. (2013). Metagenomics uncovers a new group of low GC and ultra-small marine Actinobacteria. Sci. Rep. 3, 2471. doi: 10.1038/srep02471

Giovannoni, S. J., Bibbs, L., Cho, J. C., Stapels, M. D., Desiderio, R., Vergin, K. L., et al. (2005). Proteorhodopsin in the ubiquitous marine bacterium SAR11. Nature 438, 82-85. doi: 10.1038/nature04032

Gomez-Consarnau, L., Akram, N., Lindell, K., Pedersen, A., Neutze, R., Milton, D. L., et al. (2010). Proteorhodopsin phototrophy promotes survival of marine bacteria during starvation. PLoS Biol. 8:e1000358. doi: 10.1371/journal.pbio. 1000358

Gomez-Consarnau, L., Gonzales, J. M., Coll-Llado, M., Gourdon, P., Pascher, T., Neutze, R., et al. (2007). Light stimulates growth of proteorhodopsin-containing marine Flavobacteria. Nature 445, 210-213. doi: 10.1038/nature05381

Gonzalez, J. M., Fernandez-Gomez, B., Fernandez-Guerra, A., GomezConsarnau, L., Sanchez, O., Coll-Llado, M., et al. (2008). Genome analysis of the proteorhodopsin-containing marine bacterium Polaribacter sp. MED152 (Flavobacteria). Proc. Natl. Acad. Sci. U.S.A. 105, 8724-8729. doi: 10.1073/pnas.0712027105

Gonzalez, J. M., Pinhassi, J., Fernandez-Gomez, B., Coll-Llado, M., GonzalezVelazquez, M., Puigbo, P., et al. (2011). Genomics of the proteorhodopsincontaining marine flavobacterium Dokdonia sp. strain MED134. Appl. Environ. Microbiol. 77, 8676-8686. doi: 10.1128/AEM.06152-11
Hauruseu, D., and Koblizek, M. (2012). Influence of light on carbon utilization in aerobic anoxygenic phototrophs. Appl. Environ. Microbiol. 78, 7414-7419. doi: 10.1128/AEM.01747-12

Humphrey, B., Kjelleberg, S., and Marshall, K. C. (1983). Responses of marine bacteria under starvation conditions at a solid-water interface. Appl. Environ. Microbiol. 45, 43-47.

Jacobs, S. (1965). The determination of nitrogen in biological materials. Methods Biochem. Anal. 13, 241-263.

Kimura, H., Young, C. R., Martinez, A., and Delong, E. F. (2011). Light-induced transcriptional responses associated with proteorhodopsin-enhanced growth in a marine flavobacterium. ISME J. 5, 1641-1651. doi: 10.1038/ismej.2011.36

Kirchman, D. L., and Hanson, T. E. (2013). Bioenergetics of photoheterotrophic bacteria in the oceans. Environ. Microbiol. Rep. 5, 188-199. doi: 10.1111/j.17582229.2012.00367.x

Lauro, F. M., McDougald, D., Thomas, T., Williams, T. J., Egan, S., Rice, S., et al. (2009). The genomic basis of trophic strategy in marine bacteria. Proc. Natl. Acad. Sci. U.S.A. 106, 15527-15533. doi: 10.1073/pnas.0903507106

Matallana-Surget, S., Douki, T., Cavicchioli, R., and Joux, F. (2009). Remarkable resistance to UVB of the marine bacterium Photobacterium angustum explained by an unexpected role of photolyase. Photochem. Photobiol. Sci. 8, 1313-1320. doi: $10.1039 / \mathrm{b} 902715 \mathrm{~g}$

Monod, J. (1950). La technique de culture continue: théorie et applications. Ann. Inst. Pasteur 79, 390-410.

Oh, H. M., Kwon, K. K., Kang, I., Kang, S. G., Lee, J. H., Kim, S. J., et al (2010). Complete genome sequence of "Candidatus Puniceispirillum marinum" IMCC1322, a representative of the SAR116 clade in the Alphaproteobacteria. J. Bacteriol. 192, 3240-3241. doi: 10.1128/JB.00347-10

Palovaara, J., Akram, N., Baltar, F., Bunse, C., Forsberg, J., Pedrós-Alió, C., et al. (2014). Stimulation of growth by proteorhodopsin phototrophy involves regulation of central metabolic pathways in marine planktonic bacteria. Proc. Natl. Acad. Sci. U.S.A. 111, E3650-E3658. doi: 10.1073/pnas.1402617111

Pirt, S. J. (1965). The maintenance energy of bacteria in growing cultures. Proc. $R$. Soc. Lond. B Biol. Sci. 163, 224-231. doi: 10.1098/rspb.1965.0069

Riedel, T., Gomez-Consarnau, L., Tomasch, J., Martin, M., Jarek, M., Gonzalez, J. M., et al. (2013). Genomics and physiology of a marine flavobacterium encoding a proteorhodopsin and a xanthorhodopsin-like protein. PLOS ONE 8:e57487. doi: 10.1371/journal.pone.0057487

Riedel, T., Tomasch, J., Buchholz, I., Jacobs, J., Kollenberg, M., Gerdts, G., et al. (2010). Constitutive expression of the proteorhodopsin gene by a flavobacterium strain representative of the proteorhodopsin-producing microbial community in the North Sea. Appl. Environ. Microbiol. 76, 3187-3197. doi: 10.1128/AEM.02971-09

Rusch, D. B., Halpern, A. L., Sutton, G., Heidelberg, K. B., Williamson, S., Yooseph, S., et al. (2007). The Sorcerer II Global Ocean Sampling expedition: northwest Atlantic through eastern tropical Pacific. PLoS Biol. 5:e77. doi: 10.1371/journal.pbio.0050077

Sabehi, G., Beja, O., Suzuki, M. T., Preston, C. M., and DeLong, E. F. (2004). Different SAR86 subgroups harbour divergent proteorhodopsins. Environ. Microbiol. 6, 903-910. doi: 10.1111/j.1462-2920.2004.00676.x

Sabehi, G., Loy, A., Jung, K. H., Partha, R., Spudich, J. L., Isaacson, T., et al. (2005). New insights into metabolic properties of marine bacteria encoding proteorhodopsins. PLoS Biol. 3:e273. doi: 10.1371/journal.pbio. 0030273

Steindler, L., Schwalbach, M. S., Smith, D. P., Chan, F., and Giovannoni, S. J. (2011). Energy starved Candidatus Pelagibacter ubique substitutes light-mediated ATP production for endogenous carbon respiration. PLoS ONE 6:e19725. doi: 10.1371/journal.pone.0019725

Stingl, U., Desiderio, R. A., Cho, J. C., Vergin, K. L., and Giovannoni, S. J. (2007a). The SAR92 clade: an abundant coastal clade of culturable marine bacteria possessing proteorhodopsin. Appl. Environ. Microbiol. 73, 2290-2296. doi: 10.1128/AEM.02559-06

Stingl, U., Tripp, H. J., and Giovannoni, S. J. (2007b). Improvements of highthroughput culturing yielded novel SAR11 strains and other abundant marine bacteria from the Oregon coast and the Bermuda Atlantic Time Series study site. ISME J. 1, 361-371. doi: 10.1038/ismej.2007.49

Van Wambeke, F., Catala, P., Pujo-Pay, M., and Lebaron, P. (2011). Vertical and longitudinal gradients in HNA-LNA cell abundances and cytometric characteristics in the Mediterranean Sea. Biogeosciences 8, 1853-1863. doi: 10.5194/bg-8-1853-2011 
Wang, W.-W., Sineshchekov, O. A., Spudich, E. N., and Spudich, J. L. (2003). Spectroscopic and photochemical characterization of a deep ocean proteorhodopsin. J. Biol. Chem. 278, 33985-33991. doi: 10.1074/jbc.M305716200

Wang, Z., O'Shaughnessy, T. J., Soto, C. M., Rahbar, A. M., Robertson, K. L., Lebedev, N., et al. (2012). Function and regulation of Vibrio campbellii proteorhodopsin: acquired phototrophy in a classical organoheterotroph. PLoS ONE 7:e38749. doi: 10.1371/journal.pone.0038749

Williams, T. J., Ertan, H., Ting, L., and Cavicchioli, R. (2009). Carbon and nitrogen substrate utilization in the marine bacterium Sphingopyxis alaskensis strain RB2256. ISME J. 3, 1036-1052. doi: 10.1038/ismej.2009.52
Conflict of Interest Statement: The authors declare that the research was conducted in the absence of any commercial or financial relationships that could be construed as a potential conflict of interest.

Copyright (๐ 2015 Courties, Riedel, Rapaport, Lebaron and Suzuki. This is an openaccess article distributed under the terms of the Creative Commons Attribution License $(C C B Y)$. The use, distribution or reproduction in other forums is permitted, provided the original author(s) or licensor are credited and that the original publication in this journal is cited, in accordance with accepted academic practice. No use, distribution or reproduction is permitted which does not comply with these terms. 